\title{
Optimum Power Output Control of a Wind Turbine Rotor
}

\author{
S. Wijewardana, M. H. Shaheed, and R. Vepa \\ School of Engineering and Material Science, Queen Mary University of London, London E14NS, UK \\ Correspondence should be addressed to R. Vepa; r.vepa@qmul.ac.uk
}

Received 11 December 2015; Accepted 24 January 2016

Academic Editor: Tariq Iqbal

Copyright (C) 2016 S. Wijewardana et al. This is an open access article distributed under the Creative Commons Attribution License, which permits unrestricted use, distribution, and reproduction in any medium, provided the original work is properly cited.

\begin{abstract}
An active and optimum controller is applied to regulate the power output from a wind turbine rotor. The controller is synthesized in two steps. The first step defines the equilibrium operation point and ensures that the desired equilibrium point is stable. The stability of the equilibrium point is guaranteed by a control law that is synthesized by applying the methodology of model predictive control (MPC). The method of controlling the turbine involves pitching the turbine blades. In the second step the blade pitch angle demand is defined. This involves minimizing the mean square error between the actual and desired power coefficient. The actual power coefficient of the wind turbine rotor is evaluated assuming that the blade is capable of stalling, using blade element momentum theory. This ensures that the power output of the rotor can be reduced to any desired value which is generally not possible unless a nonlinear stall model is introduced to evaluate the blade profile coefficients of lift and drag. The relatively simple and systematic nonlinear modelling and MPC controller synthesis approach adopted in this paper clearly highlights the main features on the controller that is capable of regulating the power output of the wind turbine rotor.
\end{abstract}

\section{Introduction}

Wind turbines are rotating machines that absorb energy from the wind by exploiting the aerodynamic forces of lift and drag. The lift and the drag forces act on the rotating turbine blades and the resulting kinetic energy is then transferred into the rotor and transformed into electrical energy using a generator. The rate of energy extracted from the wind is the power output of the rotor. The actual power output of a wind turbine rotor generally fluctuates and depends to a large extent on the wind speed. Generally wind turbines are installed over regions where the wind resources are high and consequently the power output not only is high but also fluctuates significantly, irrespective of its architecture. Wind turbines may be broadly classified as vertical axis wind turbines and horizontal axis wind turbines [1]. While horizontal axis wind turbines (HAWTs) can operate both as fixed and as variable speed machines, variable speed operation permits greater control on the power generated by the rotor. For most power generation applications and for feeding a power grid [2], it is essential that the power is uniformly regulated within an upper and a lower limit. Exceeding the upper limit can damage the wind turbine and/or the generator. In many high wind situations in the past, wind turbines are known to have caught fire and caused extensive damage. Low power operation of the wind turbine, under low wind conditions, is generally not economical and for this reason the wind turbine is shut down under low wind conditions. When high winds are present the wind turbine output must be regulated, up to a certain point, so the rotor delivers a constant power output to the generator. However at extremely high wind speeds, the operation of the wind turbine may be hazardous and for this reason it must be shut down. Thus when the wind turbine is always experiencing moderately high speeds continuous control and regulation of the power output are highly desirable.

In this paper, we illustrate the process of designing a wind turbine power regulation system with an active blade controller that is designed to regulate the power output from a wind turbine rotor. The controller is synthesized in two steps. In the first step, a control law is synthesized by applying the methodology of model predictive control (MPC). Although the basic system is nonlinear, we constrict a locally linear model and apply the methodology of MPC to construct a control law over a prediction window. In the second step the blade pitch angle demand is defined. While there are 
TABLE 1: Coefficients $C_{i}, i=1,2,3, \ldots, 10$, of the approximations to the power coefficient $C_{p}(\lambda, \theta)$.

\begin{tabular}{lcccc}
\hline Model & Voltolini et al. [10] & Variable pitch operation & Constant speed operation & Variable speed operation \\
\hline$C_{1}$ & 0.5176 & 0.5 & 0.44 & 0.73 \\
$C_{2}$ & 116.0 & 116.0 & 125.0 & 151.0 \\
$C_{3}$ & 0.4 & 0.4 & 0 & 0.58 \\
$C_{4}$ & 5.0 & 0 & 0 & 0.002 \\
$C_{5}$ & 0 & 0 & 0 & 2.14 \\
$C_{6}$ & 0 & 5 & 6.94 & 13.2 \\
$C_{7}$ & 21.0 & 21 & 16.5 & 18.4 \\
$C_{8}$ & 0.0068 & 0 & 0 & 0 \\
$C_{9}$ & 0.08 & 0.08 & 0 & -0.02 \\
$C_{10}$ & 0.035 & 0.035 & -0.002 & -0.003 \\
\hline
\end{tabular}

two distinct approaches to regulating the power output of a variable speed turbine by collectively controlling the pitch angle of the blades at the hub of the rotor, an alternate method based on operating the blade at an optimum angle, irrespective of the blade flow characteristics, can completely regulate the power output of the wind turbine rotor. The optimum blade pitch angle demand is found by minimizing the mean square error between the actual and desired power output. Thus the optimum blade angle is defined irrespective of whether or not the blade flow has stalled. The methodology is applied to a typical wind turbine driving a DC generator, to test the efficacy of the active controller. The simulation results show that the controller is capable of regulating the actual power output within $2 \%$ of the desired power output.

\section{Fundamental Approaches to Wind Turbine Rotor Power Regulation}

In computing the power output of a typical wind turbine rotor, it is customary to use an approximation to the power coefficient $C_{p}$ and its variation with $\lambda=\omega_{m} R / V_{\mathrm{w}}$, the ratio of the propeller tip speed with wind velocity. Approximations of $C_{p}$ as functions of $\lambda$ and the blade pitch angle $\theta$ have been published in the literature [3-5] and one such approximation [5] is shown in Figure 1. In our work this approximation is used to derive the basic control law in a later section.

The aerodynamic torque extracted by the wind turbine $T_{\mathrm{wt}}$ is related to the total power absorbed by the turbine from the wind that may, respectively, be expressed as $T_{\mathrm{wt}}=P_{\mathrm{w}} / \omega_{m}$ and $P_{\mathrm{w}}=(1 / 2) \rho\left(\pi R^{2}\right) C_{p}(\lambda, \theta) V_{\mathrm{w}}^{3}$. In the equation for the wind turbine power, $\rho$ is the density of the air at the hub of the turbine, $R$ is the rotor radius, $C_{p}(\lambda, \theta)$ is a power coefficient that is a function of $\lambda=\omega_{m} R / V_{\mathrm{w}}$, the tip speed ratio, and $V_{\mathrm{w}}$ is the wind velocity and $\theta$ is the blade collective pitch angle. The power coefficient $C_{p}(\lambda, \theta)$ is expressed as

$$
\begin{aligned}
C_{p}(\lambda, \theta)= & C_{1}\left(\frac{C_{2}}{\lambda_{i}}-C_{3} \theta-C_{4} \theta^{C_{5}}-C_{6}\right) e^{-C_{7} / \lambda_{i}} \\
& +C_{8} \lambda, \\
\frac{1}{\lambda_{i}}= & \frac{1}{\lambda+C_{9} \theta}-\frac{C_{10}}{1+\theta^{3}} .
\end{aligned}
$$

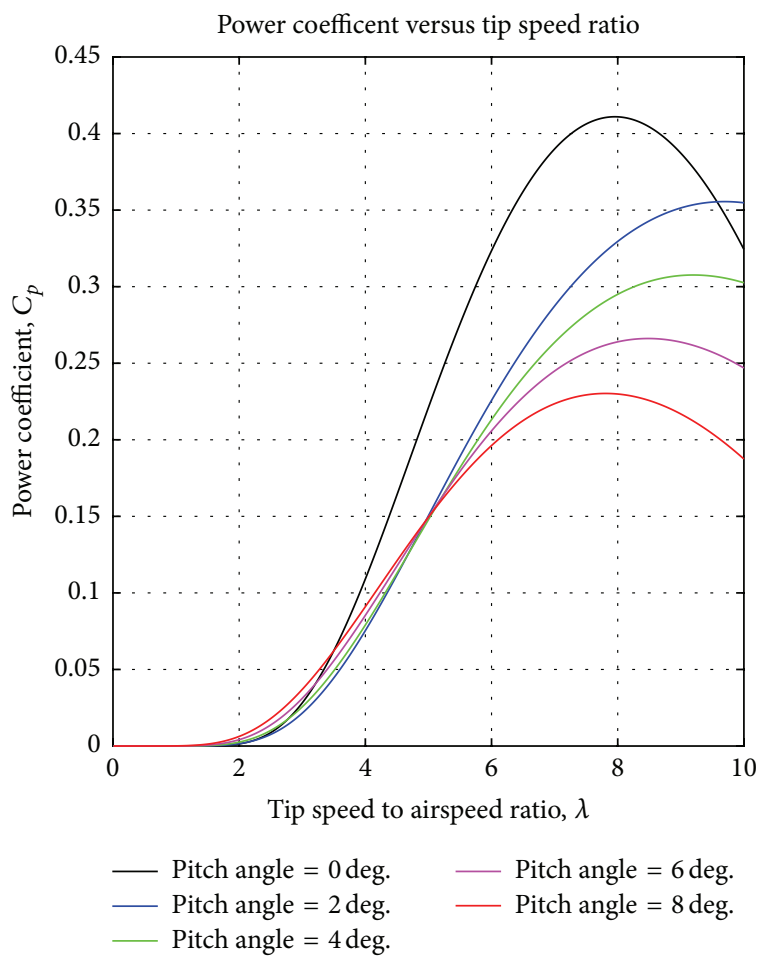

FIgURE 1: The power coefficient $C_{p}$ and its variation with $\lambda$ and the blade pitch angle.

The coefficients $C_{i}, i=1,2,3, \ldots, 10$, of the approximations to the power coefficient $C_{p}(\lambda, \theta)$ are given in Table 1 .

A close examination of Figure 1 reveals that it is possible to control the power output of the wind turbine rotor, by changing collectively the pitch angles of all of the rotor blades that are attached to the hub. Broadly, there are two different approaches of achieving this. The first approach is based on incrementally increasing the pitch angle which results in a monotonic decrease in the power coefficient for most values of the tip speed ratio $\lambda$. An alternate approach is to operate the wind turbines with blades set at an angle just below the stall angle. The stall regulated approach is recommended, amongst others, by [6,7], while [8] has reviewed a number of other techniques including advanced blade pitch control involving 
both collective and cyclic changes of blade pitch, blade twist control, variable diameter rotors, and active and passive flow control devices. Thus any increase in the blade pitch angle beyond the stall angle accentuates the occurrence of stall leading to rapid decrease in the power coefficient. While these approaches to the control of the power output delivered by a wind turbine rotor have been applied in the literature, a close examination of the requirements underscored the need for regulating the power output; this implies maintaining a steady constant output of power. However, while approximations to the power coefficient of the type presented in [5] are useful in deriving the control laws, the complete evaluation of the output power must necessarily be done by using a more extensive theory such as the blade element momentum (BEM) theory [9].

\section{Model Predictive Controller Synthesis}

Model predictive control (MPC) refers to a technique based on applying the input control over a receding horizon, in the discrete time domain. The computation of the MPC law is based on the optimization of a cost function which is often the same as the one used in discrete time optimal control over a finite time horizon. The optimization is recursive and is repeated with every new set of response measurements. Methods vary depending on the cost function employed, the type of model used for prediction of the response, and tradeoffs between prediction and control horizons. In this paper we employ a (time varying) linear model and a quadratic objective function. The basic idea is to use the model to predict the response over a finite time horizon and use the predicted response to evaluate the objective function. The objective function in minimized to obtain the control. To implement the control law at the next instant of time, the measured states at the current time are used instead of the predicted states at the next instant of time. For an excellent tutorial overview of MPC synthesis the reader is referred to [11].

To synthesize the controller we will adopt the strategy of MPC using a locally linear model of the wind turbine dynamics. To briefly describe the synthesis of linear optimal control based on the MPC approach, consider a linear discrete time system in the following form:

$$
\begin{aligned}
\mathbf{x}(k+1) & =\mathbf{A}(k) \mathbf{x}(k)+\mathbf{B}(k) \mathbf{u}(k), \\
\mathbf{y}(k) & =\mathbf{C}(k) \mathbf{x}(k) .
\end{aligned}
$$

Our aim is to find an optimal control input sequence defined over a control prediction window, $\mathbf{u}(j), j=0,1,2, \ldots, N-1$, or the vector $\mathbf{U}=\left[\begin{array}{llll}\mathbf{u}^{T}(0) & \mathbf{u}^{T}(1) & \cdots & \mathbf{u}^{T}(N-1)\end{array}\right]^{T}$ so as to minimize the performance index;

$$
\begin{aligned}
& J(\mathbf{x}(0), \mathbf{U})=\sum_{k=0}^{N-1}\left\{\mathbf{x}^{T}(k) \mathbf{Q} \mathbf{x}(k)+\mathbf{u}^{T}(k) \mathbf{R u}(k)\right\} \\
& +\mathbf{x}^{T}(N) \mathbf{Q}_{N} \mathbf{x}(N)
\end{aligned}
$$

Defining the vector, $\mathbf{X}=\left[\begin{array}{llll}\mathbf{x}^{T}(1) & \mathbf{x}^{T}(2) & \cdots & \mathbf{x}^{T}(N-\end{array}\right.$ 1) $\mathbf{x}^{T}(N)$ ], we may write

$$
J(\mathbf{x}(0), \mathbf{U})=\mathbf{X}^{T} \overline{\mathbf{Q}} \mathbf{X}+\mathbf{U}^{T} \overline{\mathbf{R}} \mathbf{U}
$$

where $\overline{\mathbf{Q}}$ is a block diagonal matrix with matrix $\mathbf{Q}$ along the diagonal except the last element which is $\mathbf{Q}_{N}$ and $\overline{\mathbf{R}}$ is a block diagonal matrix with matrix $\mathbf{R}$ along the diagonal. Using the state space model (2) recursively, we may construct a prediction model in the following form:

$$
\mathbf{X}=\mathbf{S U}+\mathbf{T} \mathbf{x}(0),
$$

where

$$
\begin{aligned}
\mathbf{S} & =\left[\begin{array}{cccc}
\mathbf{B} & \mathbf{0} & \mathbf{0} & \mathbf{0} \\
\mathbf{A B} & \mathbf{B} & \mathbf{0} & \mathbf{0} \\
\vdots & \vdots & \vdots & \vdots \\
\mathbf{A}^{N-1} \mathbf{B} & \mathbf{A}^{N-2} \mathbf{B} & \cdots & \mathbf{B}
\end{array}\right], \\
\mathbf{T} & =\left[\begin{array}{c}
\mathbf{A} \\
\mathbf{A}^{2} \\
\vdots \\
\mathbf{A}^{N}
\end{array}\right]
\end{aligned}
$$

Thus the cost function may be expressed as

$$
\begin{aligned}
J(\mathbf{x}(0), \mathbf{U})= & \frac{1}{2} \mathbf{x}(0)^{T} 2\left(\mathbf{T}^{T} \overline{\mathbf{Q}} \mathbf{T}+\mathbf{Q}\right) \mathbf{x}(0) \\
& +\frac{1}{2} \mathbf{U}^{T}\left(\overline{\mathbf{R}}+\mathbf{S}^{T} \overline{\mathbf{Q}} \mathbf{S}\right) \mathbf{U} \\
& +2 \mathbf{x}^{T}(0) \mathbf{T}^{T} \overline{\mathbf{Q}} \mathbf{S} \mathbf{U}
\end{aligned}
$$

which is written as

$$
J(\mathbf{x}(0), \mathbf{U})=\frac{1}{2} \mathbf{x}(0)^{T} \mathbf{G} \mathbf{x}(0)+\frac{1}{2} \mathbf{U}^{T} \mathbf{H} \mathbf{U}+\mathbf{x}^{T}(0) \mathbf{F U} .
$$

The optimum control sequence is obtained by setting the gradient of $J(\mathbf{x}(0), \mathbf{U})$ to zero. Minimizing the cost function results in

$$
\begin{aligned}
\frac{d J(\mathbf{x}(0), \mathbf{U})}{d \mathbf{U}} & =\mathbf{U}^{T} \mathbf{H}+\mathbf{x}^{T}(0) \mathbf{F}=0 \Longrightarrow \\
\mathbf{H U}+\mathbf{F}^{T} \mathbf{x}(0) & =0 \Longrightarrow \\
\mathbf{U} & =-\mathbf{H}^{-1} \mathbf{F}^{T} \mathbf{x}(0) .
\end{aligned}
$$

The state $\mathbf{x}(0)$, at the start of the prediction window, is assumed to represent the state at the next time instant, in real time. The control law based on the receding horizon is

$$
\mathbf{u}(k)=-\left[\begin{array}{llll}
1 & 0 & \cdots & 0
\end{array}\right] \mathbf{H}^{-1} \mathbf{F}^{T} \mathbf{x}(k) .
$$

The control sequence is recursively calculated over successive control prediction windows. To implement the controller we will need to establish a locally linear model. Thus we consider the development of a continuous time state space model of the wind turbine dynamics in the next section. 


\section{Wind Turbine Dynamic Modelling}

The basic architecture of the wind turbine is assumed to consist of a three-bladed rotor attached to a drive shaft at the rotor hub. The drive shaft drives a gear-box or drive train, the output of which drives the generator. If the wind turbine input torque is given by $T_{\mathrm{wt}}=P_{\mathrm{w}} / \omega_{m}$, the drive train load torque is assumed to be $T_{\mathrm{dwt}}$, the generator driving torque at the output of the drive train is assumed to be $T_{\text {dgen }}$, and the generator load torque is assumed to be $T_{\text {gen }}$. The static relationship between the drive train input and output torques is $T_{\text {dwt }}=N_{\text {gr }} T_{\text {dgen }}$, where $N_{\text {gr }}$ is the gear ratio of the high speed generator side speed to the wind turbine side speed. The wind turbine rotor shaft is assumed to be elastic and therefore flexible. The net shaft twist of one end relative to the other is assumed to be $\delta$. Following [12], the equations of motion of the wind turbine rotor speed $\omega_{m}$ and the generator rotor speed $\omega_{\mathrm{g}}$ are, respectively, given by

$$
\begin{gathered}
J_{r} \dot{\omega}_{m}=T_{\mathrm{wt}}-T_{\mathrm{dwt}}, \\
J_{\mathrm{g}} \dot{\omega}_{\mathrm{g}}=T_{\text {dgen }}-T_{\mathrm{gen}} .
\end{gathered}
$$

The shaft twist dynamics are modelled as

$$
T_{\mathrm{dwt}}=B_{\mathrm{sh}} \dot{\delta}+K_{\mathrm{sh}} \delta
$$

where $B_{\text {sh }}$ and $K_{\text {sh }}$ are the viscous damping and elastic stiffness of the shaft, respectively. The twist rate $\dot{\delta}$ is related to the shaft speeds by the following relation:

$$
\dot{\delta}=\omega_{m}-\frac{\omega_{\mathrm{g}}}{N_{\mathrm{gr}}} .
$$

The tower is assumed to be an elastic column and its secondorder dynamics are represented by a single vibration mode satisfying

$$
m_{\mathrm{tw}} \ddot{\xi}+b_{\mathrm{tw}} \dot{\xi}+k_{\mathrm{tw}} \xi=f_{\mathrm{tw}}
$$

where $m_{\mathrm{tw}}, b_{\mathrm{tw}}, k_{\mathrm{tw}}$, and $f_{\mathrm{tw}}$ are the generalised mass, generalised damping, generalised stiffness, and generalised disturbing force acting on the tower in the assumed mode. The blade pitch angle actuator is represented by the secondorder dynamics given by

$$
\ddot{\theta}+2 \zeta_{b} \omega_{n b} \dot{\theta}+\omega_{n b}^{2} \theta=\omega_{n b}^{2} \theta_{c},
$$

where $\omega_{n b}$ is the actuator natural frequency, $\zeta_{b}$ is the damping ratio of the blade actuator, and $\theta_{c}$ is the blade angle control input which may be expressed in terms of error between the blade angle feedback and the demanded blade angle as $e=\theta-\theta_{d}$. Finally the generator torque dynamics are modelled as a first-order lag and are given by

$$
\tau_{\text {gen }} \dot{T}_{\text {gen }}+T_{\text {gen }}=T_{\text {g,ref }},
$$

where $\tau_{\text {gen }}$ is the time constant of the generator's firstorder lag dynamics and $T_{\text {g,ref }}$ is the generator reference output. Equations (11)-(16) may be linearised and expressed in state space form, with one control input $\left(\theta_{c}\right)$, one reference command input $\left(T_{\mathrm{g}, \mathrm{ref}}\right)$, and one disturbance input $\left(f_{\mathrm{tw}}\right)$. The wind speed $V_{\mathrm{w}}$ is also assumed to include a disturbance component. The state vector comprises $\omega_{m}, \omega_{\mathrm{g}}, \delta, \xi, \dot{\xi}, \theta, \dot{\theta}$, and $T_{\text {gen }}$. To determine $\mathbf{A}(k)$ and $\mathbf{B}(k)$ matrices, the equations of motion and the expression for the power coefficient are carefully linearised at each sampling instant and the linearised continuous time equations are converted to discrete time.

\section{Blade Element Momentum Theory for Power Coefficient}

The calculation of the power output must be done with some care using the BEM theory as outlined in [9]. In [9] based on BEM theory it is shown that the power coefficient may be expressed as

$$
C_{p}=\frac{P_{\mathrm{w}}}{P_{\text {wind }}}=\frac{1}{\lambda_{f}} \int_{\bar{r}_{h}}^{1} \frac{\sigma^{\prime}(1-a)^{2}}{\sin \phi}\left(\frac{C_{l}}{\tan \phi}+C_{d}\right) \bar{r}^{2} d \bar{r} .
$$

Hence the axial induction factor $a$ may be expressed as

$$
1-a=\left\{1+\frac{\sigma^{\prime}}{8 Q_{\text {tip }} \sin \phi}\left(\frac{C_{l}}{\tan \phi}+C_{d}\right)\right\}^{-1} .
$$

In the above equations for the power coefficient $C_{p}$ and for the axial induction factor $a, \phi$ is the inflow angle defined of the in-plane and tangential velocity components as

$$
\phi=\tan ^{-1}\left(\frac{u_{P}}{u_{T}}\right)=\tan ^{-1}\left(\frac{\lambda_{f}(1-a)}{\bar{r}\left(1+a^{\prime}\right)}\right),
$$

where $\lambda_{f}$ is the inflow ratio, $\lambda_{f}=1 / \lambda=V_{\mathrm{w}} / \omega_{m} R, Q_{\text {tip }}$ is Prandtl's tip-loss correction factor which will be evaluated from the expression in [9], $\sigma^{\prime}$ is the local solidity ratio for a $N$-bladed rotor given in terms of the blade chord $c$ and the local radial position $r$ by $\sigma^{\prime}=N c / \pi r$, and $a^{\prime}$ is the angular velocity induction factor given by

$$
a^{\prime}=\frac{\sigma^{\prime}(1-a)}{8 Q_{\text {tip }}\left(\sin ^{2} \phi\right)} \lambda_{f}\left(-C_{l} \sin \phi+C_{d} \cos \phi\right) .
$$

The sectional angle of attack is $\alpha=\phi-\theta$ where $\theta$ is blade section pitch angle. Thus to evaluate the power coefficient $C_{p}$, the sectional lift and drag coefficients $C_{l}$ and $C_{d}$ must be known. These two coefficients are evaluated both for the case of stalled blade and for the case without stall as described in the next section.

\section{Dynamic Stall Modelling}

According to the BEM theory, aerodynamic loads on a section of an aerofoil are proportional to the dynamic pressure at only that section. Lift and drag coefficients are proportionality constants that enable the calculation of aerodynamic forces. Spera [13] has provided several empirical methods for estimating the sectional lift and drag coefficients, $C_{l}$ and $C_{d}$ for several aerofoil sections, both for the case of stalled blade and for the case without stall. For the case without stall, 
TABLE 2: Typical parameter and initial state values for simulation.

\begin{tabular}{lccc}
\hline Parameter & Primary value & State/input & Initial value \\
\hline$C_{p-\text { nom }}$ & 0.38 & $V_{\mathrm{w}}$ & $8 \mathrm{~m} / \mathrm{s}$ \\
$R$ & $63 \mathrm{~m}$ & $N_{\mathrm{gr}}$ & 97 \\
$R_{\text {root }}$ & $9.3564 \mathrm{~m}$ & $\omega_{n b}$ & 0.88 \\
$J_{r}$ & $5.9154 \times 10^{7} \mathrm{kgm}^{2}$ & $\zeta_{b}$ & 0.9 \\
$J_{\mathrm{g}}$ & $500 \mathrm{kgm}^{2}$ & $\tau_{\mathrm{gen}}$ & 0.1 \\
$m_{\mathrm{tw}}$ & $4.2278 \times 10^{5} \mathrm{~kg}$ & $\omega_{\mathrm{g} \text { nom }}$ & $121.91 \mathrm{rad} / \mathrm{s}$ \\
$b_{\mathrm{tw}}$ & $2021.3 \mathrm{Nms} / \mathrm{rad}$ & $\omega_{r_{-} \text {nom }}$ & $1.26 \mathrm{rad} / \mathrm{s}$ \\
$k_{\mathrm{tw}}$ & $1.6547 \times 10^{6} \mathrm{Nm} / \mathrm{rad}$ & $N$ & 3 \\
$B_{\mathrm{sh}}$ & $8.3478 \times 10^{7} \mathrm{Nms} / \mathrm{rad}$ & $H$ & $90 \mathrm{~m}$ \\
$K_{\mathrm{sh}}$ & $8.7354 \times 10^{8} \mathrm{Nm} / \mathrm{rad}$ & $n_{\text {elements }}$ & 17 \\
\hline
\end{tabular}

the corrections are made to the two-dimensional lift curve slope, mainly for the finite aspect ratio and the finite thickness to chord ratio. For aspect ratios that are very large, the aspect ratio corrections may be ignored. For the case of the poststall flows, the models and corrections are based on the work of Viterna and Corrigan [14], Tangler and Kocurek [15], and Tangler and Ostowari [16]. In our work the blades have a very large aspect ratio and for this reason these corrections were ignored. However for the poststall case aspect ratio corrections were deemed to be important in the poststall aerofoil characteristics and therefore we apply the corrections of [14] which have also been presented by $[15,16]$. For BEM, the Viterna method provides a convenient global approach to relate the poststall $C_{l}$ and $C_{d}$ to the overall blade geometry rather than to the individual blade stations.

\section{Application to Power Output Regulation}

For purposes of power output regulation we estimate the actual power output based on the BEM theory. Then the pitch angle command is adjusted so as to minimize the mean square error between the desired power output and the actual power output. The pitch angle command is updated and the simulation over the next time step is carried out.

The simulation is begun with the open loop simulation of (11)-(16). The steady state equilibrium conditions and states are established. The local linear dynamic model is then established at each equilibrium state and the matrix coefficients of the local linear dynamic model are used to compute the model predictive control law. The control gains are computed from (10) and the controller is implemented as a receding horizon controller. Thus the control law is applied at the next sampling instant. The full nonlinear closed loop dynamics are simulated. The demanded pitch angle is then estimated using the BEM theory for the power coefficient, so as to restrict the power to fixed value.

\section{Typical Simulations and Results}

The parameters of a typical wind turbine and the blades are listed in Table 2. The time step for the computations is $\Delta t=$ $0.002 \mathrm{~s}$. At each time step the MPC law is obtained over a prediction window of 20 time steps. In the first instance, we

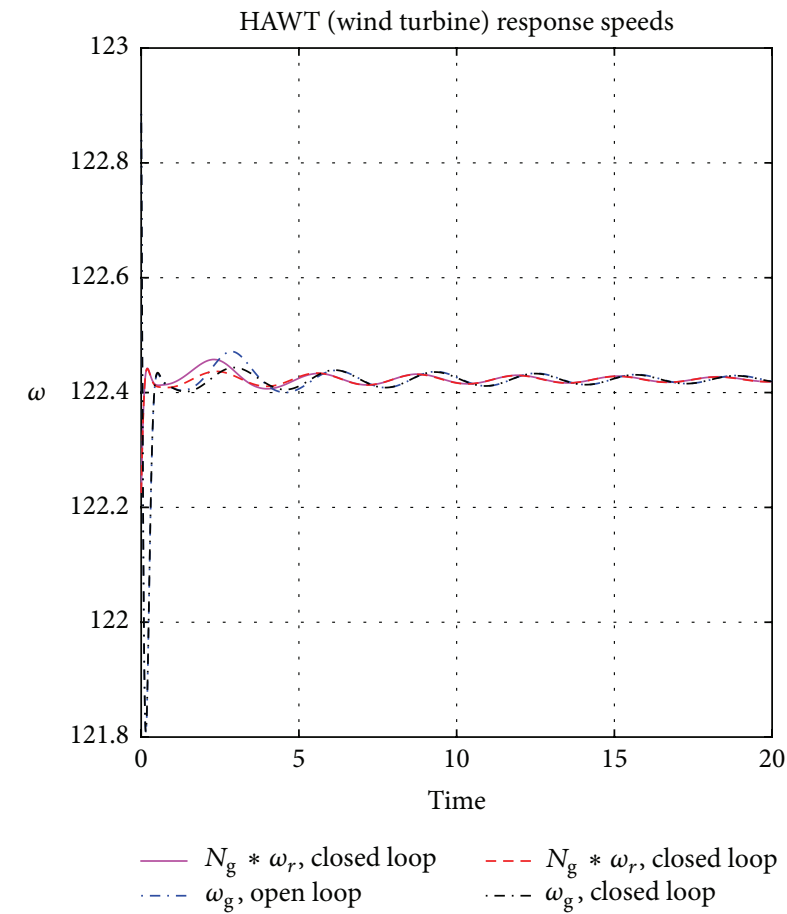

FIGURE 2: Horizontal axis wind turbine (HAWT) open and closed loop response speeds.

consider the wind turbine response speeds which are shown in Figure 2. In Figure 3 the wind turbine response torque in both the open and closed loop cases is shown. In Figure 4 the rotor twist rate corresponding to Figures 2 and 3 is shown. In these cases the demanded blade angle is set to $\theta_{d}=0^{\circ}$ so as to verify the closed loop stability.

In Figures 5 and 6 the power output and the demanded blade angle when the power is restricted in the closed loop to a prescribed limiting value are shown. The prescribed limit was set at $1200 \mathrm{Kw}$. When the limit is reduced to $700 \mathrm{Kw}$ the corresponding response curves for the power output and the demanded blade angle are shown in Figures 7 and 8. The demanded blade angle in both Figures 6 and 8 is oscillatory about a mean value. The lower the limit set for the demanded power output the higher the mean value of the demanded blade angle. 


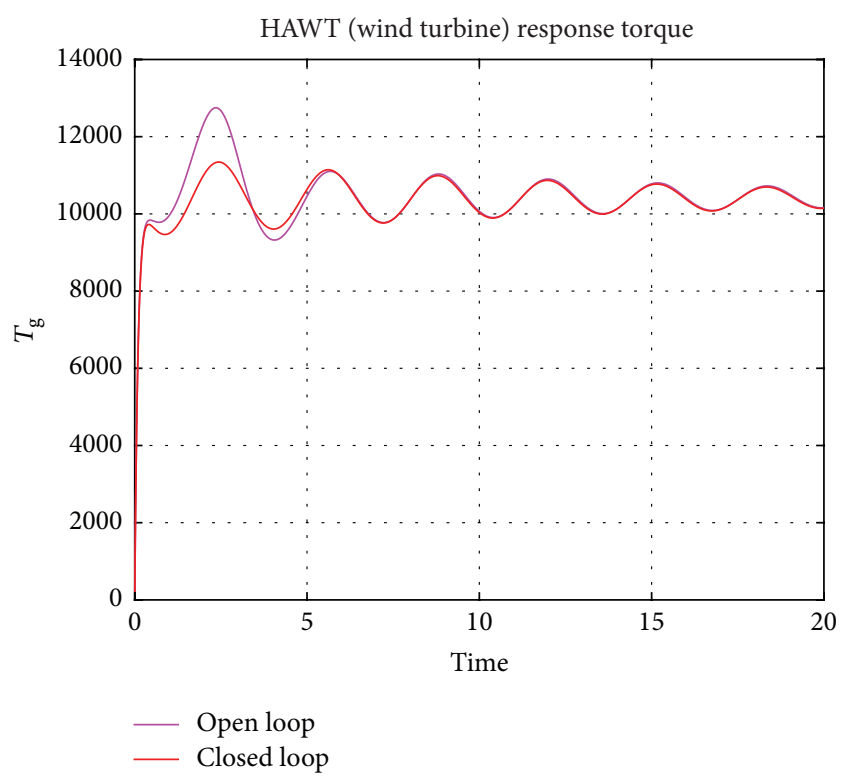

FIgURE 3: Horizontal axis wind turbine (HAWT) open and closed loop torque response.

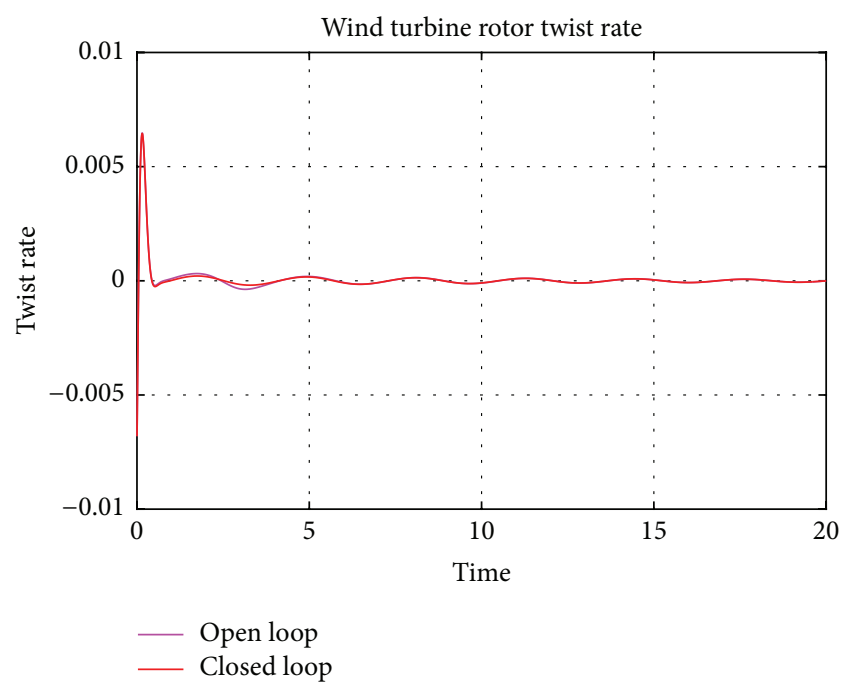

Figure 4: Horizontal axis wind turbine (HAWT) open and closed loop rotor twist rate response.

It is indeed instructive to investigate the blade angle required to completely shut down the power output from the turbine. The power output response and the corresponding demanded blade angle are shown in Figures 9 and 10, respectively. The demanded blade angle is a constant and equal to $\theta_{d}=11.6393^{\circ}$. The blade angle will now be limited to a maximum of $\theta_{d}=11^{\circ}$. The corresponding power output response and the demanded blade angle are shown in Figures 11 and 12 , respectively. While the blade angle is indeed limited to $\theta_{d}=11^{\circ}$, it may be observed that there is indeed a small residual power output of about $200 \mathrm{Kw}$.

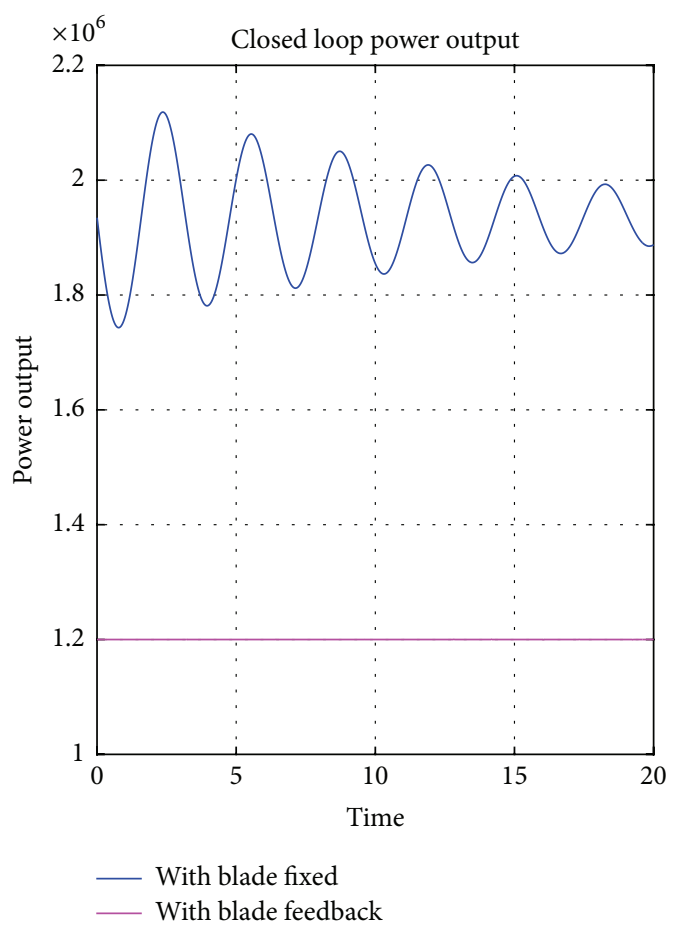

FIGURE 5: The open and closed loop power output when it is restricted in the closed loop to a prescribed limit.

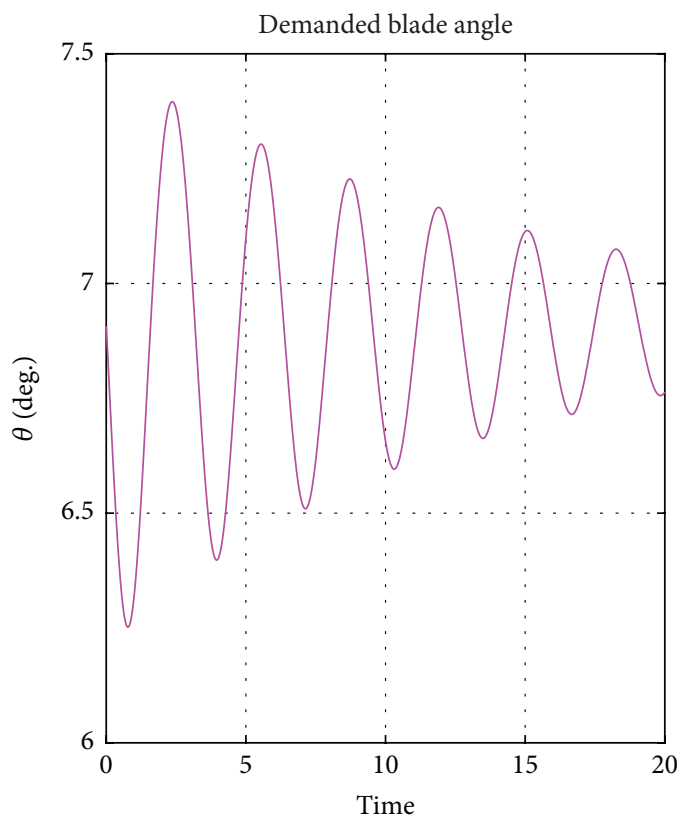

Figure 6: The open and closed loop demanded blade angle corresponding to Figure 5.

\section{Conclusions}

It is observed, from the responses shown in the figures, that the controller has all the characteristics of an active stall flow controller in the sense that the blade angle has to be increased beyond a critical value so the flow is stalled, to limit the 


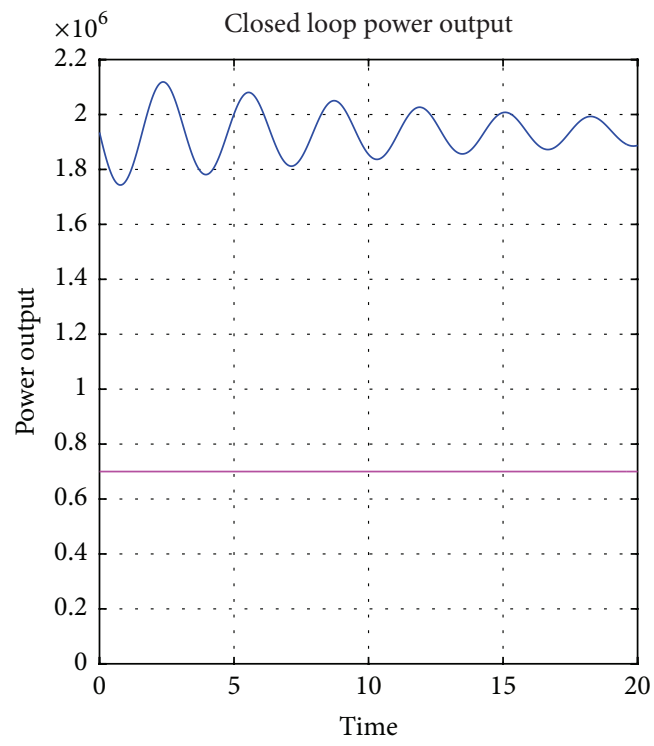

_ With blade fixed
_ With blade feedback

FIGURE 7: Response curves for the power output with the prescribed limit set at $700 \mathrm{Kw}$.

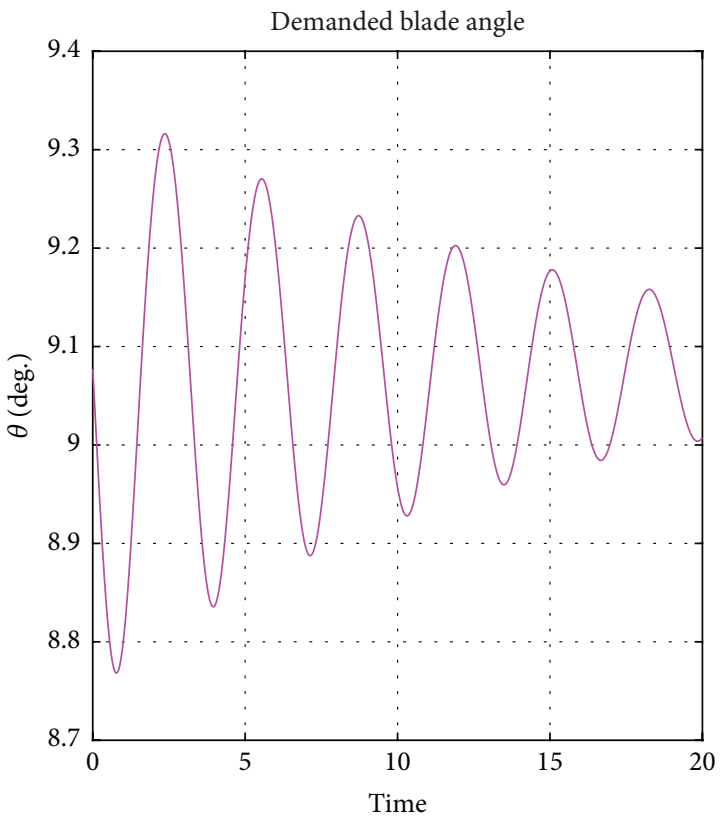

FIGURE 8: Demanded blade angle corresponding to Figure 7.

power output at zero. Although we have not used the stall flow conditions explicitly, they have been implicitly employed.

Whenever the blade section angle of attack at any blade section, which is given by $\alpha=\phi-\theta$, where $\phi$ is the inflow angle defined by (19) and $\theta$ is the blade section pitch angle, exceeds the local stall angle of attack, stall flow conditions are assumed for calculating the power coefficient $C_{p}$. These conditions depend on the blade section being considered in the evaluation of $C_{p}$ by integration of (17). (In evaluating $C_{p}$ using (17) we employed an appropriate distribution of the

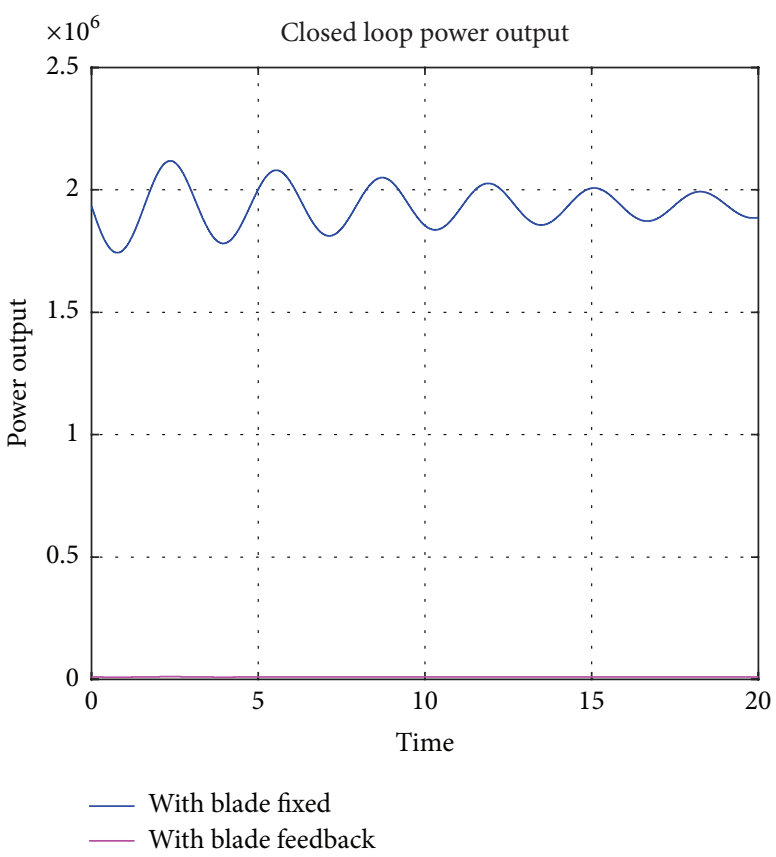

FIGURE 9: Response curves for the power output with the prescribed limit set at $0 \mathrm{Kw}$.

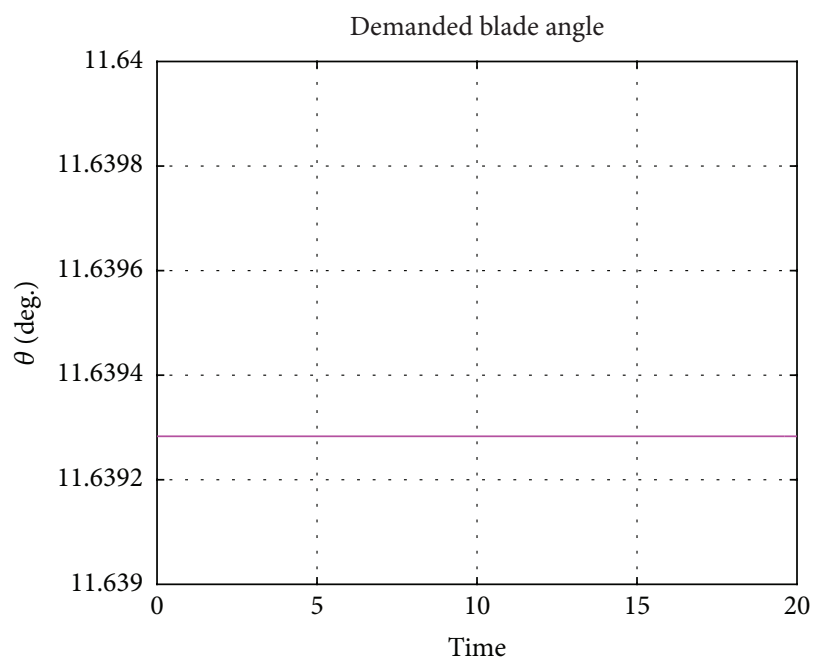

FIgURE 10: Demanded blade angle in degrees corresponding to Figure 9.

local blade chord and the blade twist or the aerofoil inlet angle along the blade length.) Hence our optimum demanded blade angle based controller, obtained by minimizing the mean square error between the actual power output and desired power output, resembles an active stall flow controller. The MPC law, used as part of the controller, has the structure of a full state feedback controller except that the feedbacks involving the blade angle and blade angle rate are interpreted as the feedbacks involving $e=\theta-\theta_{d}$ and $\dot{e}$. Thus we have provided a new and practical method of synthesizing an active stall flow controller, where the feedback control law is derived by applying the concept of MPC. 


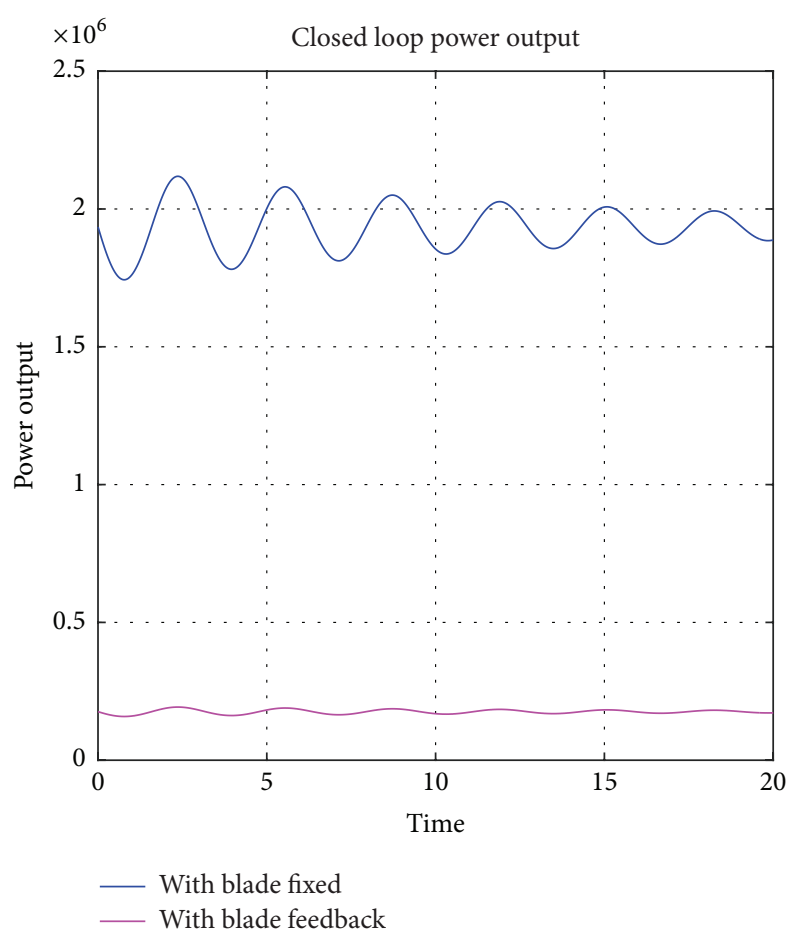

FIGURE 11: Response curves for the power output with the prescribed limit set at $0 \mathrm{Kw}$ and the blade angle limited to $\theta_{d}=11^{\circ}$.

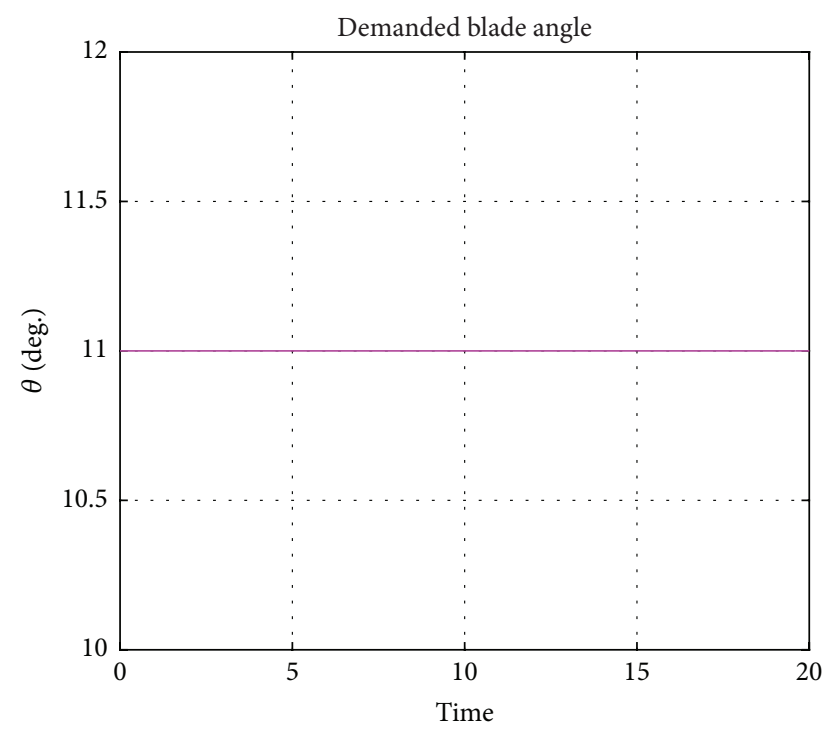

Figure 12: Demanded blade angle in degrees corresponding to Figure 11.

\section{Conflict of Interests}

The authors declare that there is no conflict of interests regarding the publication of this paper.

\section{References}

[1] T. Burton, D. Sharpe, N. Jenkins, and E. Bossanyi, Wind Energy Handbook, John Wiley \& Sons, London, UK, 2001.
[2] R. Vepa, "Nonlinear, optimal control of a wind turbine generator," IEEE Transactions on Energy Conversion, vol. 26, no. 2, pp. 468-478, 2011.

[3] P. Rosas, Dynamic influences of wind power on the power system [Ph.D. dissertation], Ørsted Institute, Section of Electric Power Engineering, Technical University of Denmark, 2003.

[4] J. G. Slootweg, S. W. H. de Haan, H. Polinder, and W. L. Kling, "General model for representing variable speed wind turbines in power system dynamics simulations," IEEE Transactions on Power Systems, vol. 18, no. 1, pp. 144-151, 2003.

[5] J. G. Slootweg, H. Polinder, and W. L. Kling, "Reduced-order modelling of wind turbines," in Wind Power in Power Systems, T. Ackermann, Ed., vol. 25, chapter 25, John Wiley \& Sons, Chicester, UK, 2005.

[6] E. Muljadi, K. Pierce, and P. Migliore, Control Strategy for Variable-Speed, Stall-Regulated Wind Turbines, NREL/CP-50024311-UC Category: 1211, National Renewable Energy Laboratory, Golden, Colo, USA, 1998.

[7] E. Muljadi, K. Pierce, and P. Migliore, "A conservative control strategy for variable-speed stall-regulated wind turbines," in Proceedings of the ASME Wind Energy Symposium, NREL/CP500-24791, pp. 91-98, National Renewable Energy Laboratory, Reno, Nev, USA, January 2000.

[8] S. J. Johnson, C. P. van Dam, and D. E. Berg, "Active load control techniques for wind turbines," Sandia Report SAND2008-4809, Sandia National Laboratories, Albuquerque, NM, USA, 2008.

[9] R. Vepa, "Dynamic modelling simulation and control of energy generation," in Wind Power Generation and Control, vol. 20 of Lecture Notes in Energy, chapter 4, Springer, London, UK, 2013.

[10] H. Voltolini, M. H. Granza, J. Ivanqui, and R. Carlson, "Modeling and simulation of the Wind Turbine Emulator using induction motor driven by torque control inverter," in Proceedings of the 10th IEEE/IAS International Conference on Industry Applications (INDUSCON 12), pp. 1-6, IEEE, Fortaleza, Brazil, November 2012.

[11] J. B. Rawlings, "Tutorial overview of model predictive control," IEEE Control Systems Magazine, vol. 20, no. 3, pp. 38-52, 2000.

[12] A. Jain, G. Schildbach, L. Fagiano, and M. Morari, "On the design and tuning of linear model predictive control for wind turbines," Renewable Energy, vol. 80, pp. 664-673, 2015.

[13] D. A. Spera, "Models of lift and drag coefficients of stalled and unstalled airfoils in wind turbines and wind tunnels," Tech. Rep. NASA/CR-2008-215434, National Aeronautics and Space Administration, Glenn Research Center, Cleveland, Ohio, USA, 2008.

[14] L. A. Viterna and R. D. Corrigan, "Fixed pitch rotor performance of large horizontal axis wind turbines," in Proceedings of the DOE/NASA Workshop on Large Horizontal Axis Wind Turbines, Cleveland, Ohio, USA, July 1981.

[15] J. L. Tangler and J. D. Kocurek, "Wind turbine post-stall airfoil performance characteristics guidelines for blade-element momentum methods," in Proceedings of the 43rd AIAA Aerospace Sciences Meeting and Exhibit, NREL/CP-500-36900, Reno, Nev, USA, January 2005.

[16] J. L. Tangler and C. Ostowari, "Horizontal axis wind turbine post stall airfoil characteristics synthesization," in Presented at the DOE/NASA Wind Turbine Technology Workshop, SERI/TP257-4400, UC Category 261, DE91002198, Cleveland, Ohio, USA, May 1984. 


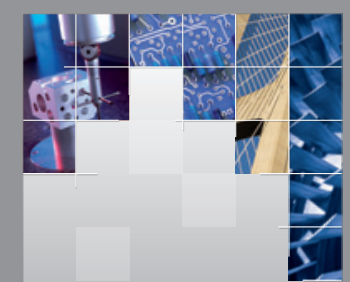

\section{Enfincering}
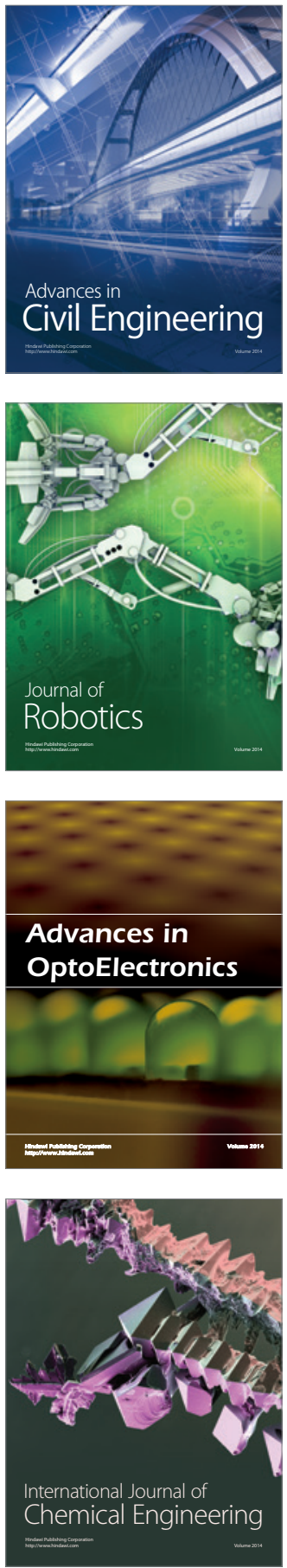

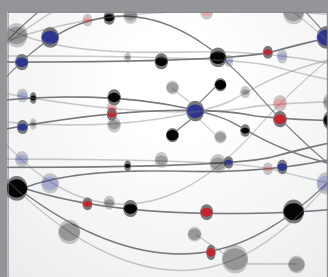

The Scientific World Journal

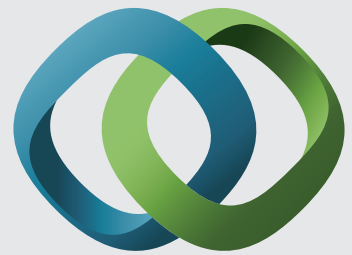

\section{Hindawi}

Submit your manuscripts at

http://www.hindawi.com
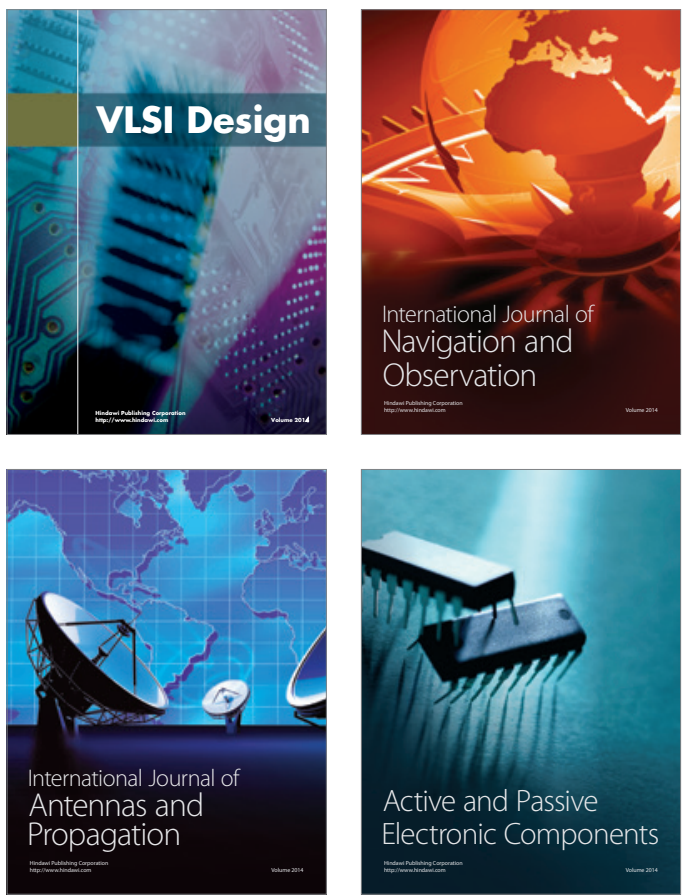
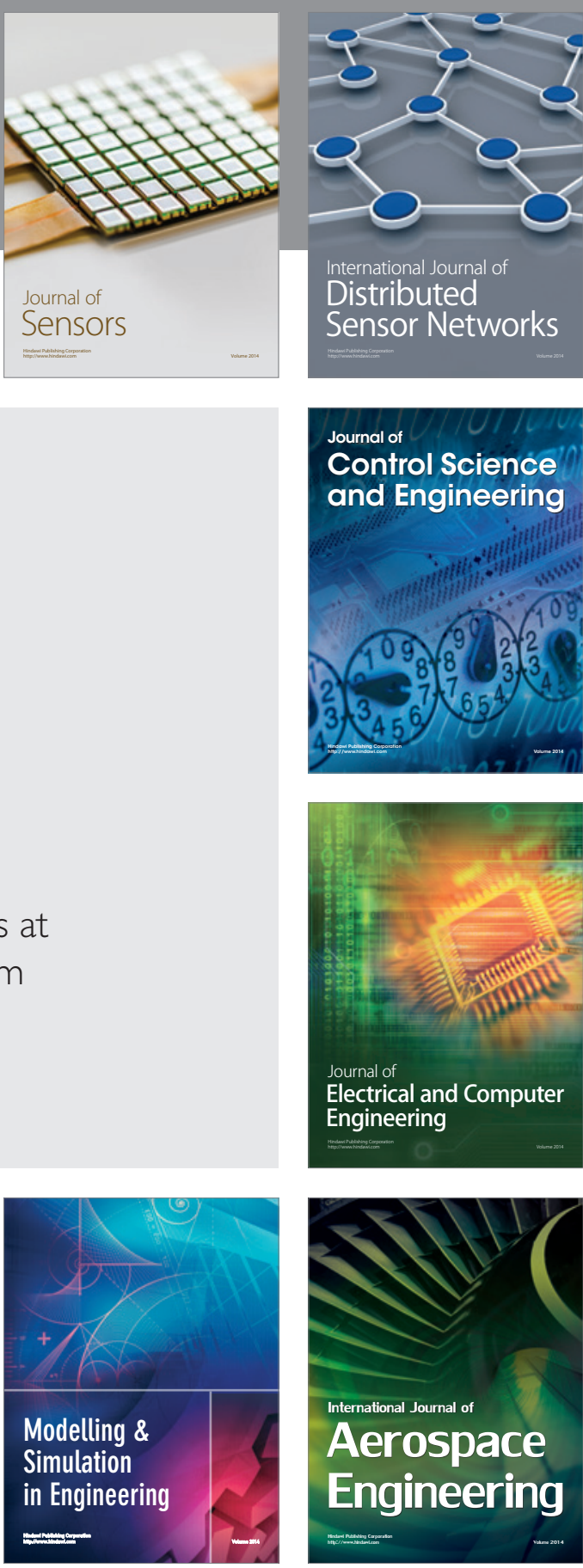

International Journal of

Distributed

Sensor Networks

Journal of

Control Science

and Engineering
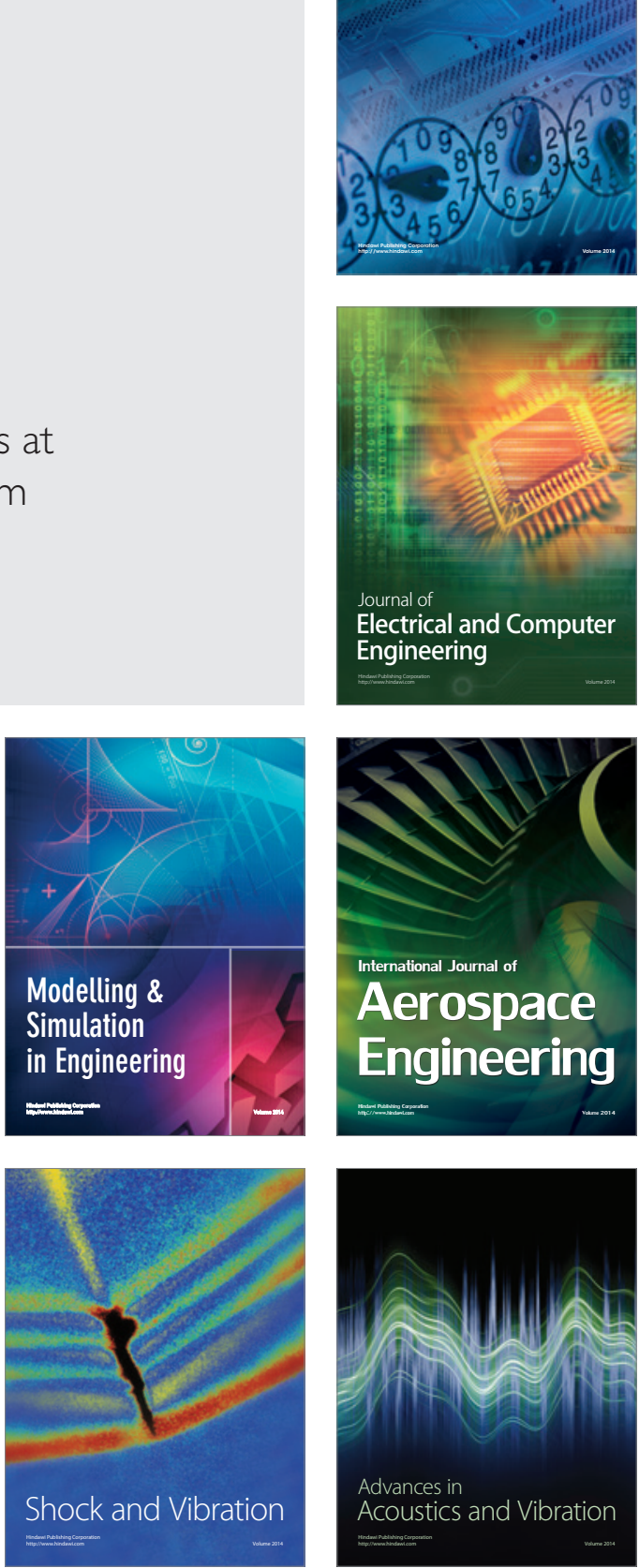